\title{
Icariin enhances cell survival in lipopolysaccharide-induced synoviocytes by suppressing ferroptosis via the Xc-/GPX4 axis
}

\author{
HUASONG LUO $^{1}$ and RUI ZHANG ${ }^{2}$ \\ ${ }^{1}$ Department of Orthopedics, The First People's Hospital of Jingzhou (First Affiliated Hospital of Yangtze University), \\ Jingzhou, Hubei 434000; ${ }^{2}$ Department of Orthopedics, Gansu Provincial Hospital of Traditional Chinese Medicine, \\ Lanzhou, Gansu 730050, P.R. China
}

Received May 25, 2020; Accepted September 16, 2020

DOI: $10.3892 / \mathrm{etm} .2020 .9504$

\begin{abstract}
The mechanism of action of synovitis, as the vital pathological process of rheumatoid arthritis and osteoarthritis, remains to be elucidated. The effects and the mechanism of icariin (ICA), which is a promising therapeutic agent in synovitis, was investigated in the present study. In addition, ferroptosis, a vital cell process involved in several diseases, was also studied in synovitis for the first time. Lipopolysacc haride (LPS)-induced synoviocytes served as a synovitis cell model. The cells were divided into control, LPS and experimental groups and were treated with different concentrations of ICA. Cell viability was determined by Cell Counting Kit- 8 assay and cell death was determined by flow cytometry. The expression levels of proteins (GPX4, SLC7A11, SLC3A2L, TRF, Nrf2 and NCOA4) were measured by western blotting. Quantification of malondialdehyde (MDA), iron and glutathione peroxidase 4 (GPX4) activity levels were performed via using corresponding assay kits. Cell death was increased, and cell viability was decreased in LPS-induced synoviocytes. Furthermore, MDA levels and iron content were elevated and GPX levels was reduced in LPS-induced synoviocytes. Transferrin receptor protein 1 and nuclear receptor coactivator 4 were upregulated and proteins of the Xc-/GPX4 axis, as well as nuclear factor erythroid 2-related factor 2, were decreased by LPS treatment. All aforementioned LPS affects were alleviated by ICA via a concentration-dependent manner. ICA counteracted the effects of RSL3, a ferroptosis activator, on cell viability, lipid peroxidation, iron content and relative protein expression of ferroptosis in synoviocytes. ICA protects the cells from death in synoviocytes induced by LPS, via the inhibition of ferroptosis by activating the Xc-/GPX4 axis, which can be exploited as a new therapeutic strategy for synovitis.
\end{abstract}

Correspondence to: Dr Rui Zhang, Department of Orthopedics, Gansu Provincial Hospital of Traditional Chinese Medicine, 418 Guazhou Road, Qilihe, Lanzhou, Gansu 730050, P.R. China E-mail: zhangruizrr@163.com

Key words: icariin, synovitis, ferroptosis, Xc-/glutathione peroxidase 4 axis, synoviocytes

\section{Introduction}

Rheumatoid arthritis is a chronic inflammatory disease caused by the dysregulation of the autoimmune system, which severely threatens the individual's health (1). Approximately $1 \%$ of the population suffers from rheumatoid arthritis worldwide, placing a heavy burden on economy and health (2). Chronic inflammation in rheumatoid arthritis leads to synovial hyperplasia and serious damage in the joints and cartilage (3). Rheumatoid arthritis is confirmed as a progressive disease and is also able to induce numerous implications on osteoporosis, myocarditis, neuropathy and pleurisy (4-6). Genetic and environmental factors are considered as the major contributors to rheumatoid arthritis $(7,8)$. Furthermore, the exact mechanism of the pathogenesis and progression of rheumatoid arthritis remains unknown (9). The major characteristic of rheumatoid arthritis is synovitis (10). Normal synovium mainly consists of fibroblast-like synoviocytes and acts as a nutrient supplier for articular cartilage and protector of joint structures or adjacent tissues (10). The number of synovial cells are significantly increased in rheumatoid arthritis and are capable of causing destruction in the joint via the release of pro-inflammatory factors $(11,12)$. In addition, synovitis is also involved in osteoarthritis. During osteoarthritis, stromal vascularization, fibrosis and hyperplasia occur in the synovium (13). Synovitis is closely associated with dysfunction and damage of the joint, as well as pain, and also contributes to the degeneration of cartilage in osteoarthritis (14). Therefore, it is important to find an effective therapeutic agent for synovitis and elucidate its mechanism, which will be beneficial for both the treatment of rheumatoid arthritis and osteoarthritis.

Icariin (ICA) as a bioactive monomer extracted from Epimedium in the Berberidaceae family, which exerts anticancer, antiaging, neuroprotective and anti-inflammation effects (15-18). ICA is confirmed as a promising agent for the treatment of rheumatoid arthritis and osteoarthritis in several previous studies. ICA was reported to inhibit osteoarthritis via suppressing pyroptosis mediated by NLRP3 (19). ICA repressed the death and angiogenesis of chondrocytes in a rat osteoarthritis model via the modulation of the TDP-43 signaling pathway (20), enhanced chondrocyte viability via promoting anaerobic glycolysis and the expression of hypoxia-inducible factor-1 $\alpha$ (21), exerted an immune-suppressive role in rheumatoid arthritis (22) 
and ameliorated rheumatoid arthritis in in a murine model of rheumatoid arthritis (23). However, the mechanism of ICA in rheumatoid arthritis and osteoarthritis is not fully elucidated, and clarifying the concrete mechanism is beneficial for developing more therapeutic drugs based on this compound.

Synovitis, as the crucial pathological process in rheumatoid arthritis and osteoarthritis, is a vital research topic. During synovitis, oxidative stress and lipid peroxidation are all involved and are confirmed as vital contributors to the pathological progress (24-26). Moreover, oxidative stress and lipid peroxidation are two major causes for ferroptosis, which is an iron-dependent, non-apoptotic form of cell death, characterized by the intracellular accumulation of reactive oxygen species. Thus, it is speculated that ferroptosis is a crucial process for injury in synovitis (27). Simultaneously, ICA has a regulatory role on iron metabolism (28). Therefore, the treatment effects of ICA may be related to the modulation of ferroptosis. In the present study, LPS-induced synovitis served as the cell model and the association between ICA and ferroptosis was investigated in the cell model of synovitis.

\section{Materials and methods}

Cell culture and treatment. Human synoviocytes (HUM-CELL-0060; Wuhan PriCells Biomedical Technology Co., Ltd.) were cultured in DMEM (Thermo Fisher Scientific, Inc.) supplemented with $1 \%$ streptomycin-penicillin, $2 \mathrm{mM}$ L-glutamine and 10\% FBS (Gibco; Thermo Fisher Scientific, Inc.) in an incubator with $5 \% \mathrm{CO}_{2}$ at $37^{\circ} \mathrm{C}$. Cells at $>80 \%$ confluence were treated with LPS $(1 \mu \mathrm{g} / \mathrm{ml}$; Sigma-Aldrich; Merck KGaA) for $24 \mathrm{~h}$ to establish a synovitis cell model as previously described (29). The cells were treated with LPS for $24 \mathrm{~h}$, followed by pretreatment with 2 (low), 5 (mid) or $10 \mu \mathrm{M}$ (high) ICA for $24 \mathrm{~h}$. Cells with no treatment served as the control group. Next, in order to further investigate the potential mechanism of action of ICA, RSL3 was used to induce ferroptosis in synoviocyte. We pre-treated synoviocyte with RSL3 (1 $\mu \mathrm{M}$, cat. no. HY-100218A, MedChemExpress, China) for $8 \mathrm{~h}$. After that, cells were incubated with ICA for $24 \mathrm{~h}$ and used for further experiments.

Measurement of cell death. Following LPS and ICA treatment, cells were digested by trypsin $(0.25 \%)$ and resuspended in binding buffer. Subsequently, the cells were incubated with Annexin V-FITC for $15 \mathrm{~min}$ at $37^{\circ} \mathrm{C}$ in the dark. Subsequently, propidium iodide was added into the cells for 2 min at $37^{\circ} \mathrm{C}$ before measurement. Flow cytometry (BD FACSCanto II; BD Biosciences) was used for cell death measurement. The apoptotic cells were analyzed using a FlowJo ${ }^{\mathrm{TM}}$ v10.7 software (Becton-Dickinson \& Company). The levels of cell death were calculated using the formula: Cell death ratio=Q1 + Q2 + Q3.

Quantification of malondialdehyde (MDA), iron and glutathione peroxidase 4 (GPX4) activity levels. The levels of MDA, iron and GPX4 activity were determined using their corresponding kits. MDA was detected via using the Lipid Peroxidation (MDA) Assay kit (cat. no. ab118970; Abcam), iron was determined by using the Iron Assay kit (cat. no. ab83366; Abcam) and GPX4 was detected using the GPX activity detection kit (cat. no. BC1195; Beijing Solarbio Science \&
Technology Co., Ltd.). All detection processes were performed according to the manufacturer's protocol.

Western blotting. The cells were harvested after treatment and, following lysis in NP40 buffer (Beijing Solarbio Science \& Technology Co., Ltd.), the supernatant was centrifuged at $4^{\circ} \mathrm{C}$ at $12,000 \mathrm{x}$ g for $15 \mathrm{~min}$ for collecting the samples. The BCA protein Assay kit (cat. no. P0012S; Beyotime Institute of Biotechnology) was used to quantify protein samples. Subsequently, separation of the total protein was performed on $10 \%$ SDS-PAGE. Next, the proteins were transferred to polyvinyl difluoride membranes (Bio-Rad Laboratories, Inc.). Following blocking with 5\% skim milk at room temperature for $1 \mathrm{~h}$, the membranes were incubated with primary antibodies against GPX4 (1:10,000, cat. no. ab125066; Abcam), cystine/glutamate transporter (1:10,000, SLC7A11; cat. no. ab37185; Abcam), 4F2 cell-surface antigen heavy chain (1:1,000, SLC3A2L; cat. no. ab215952; Abcam), transferrin receptor protein (1:1,000, TFR; cat. no. ab84036; Abcam), nuclear factor erythroid 2-related factor 2 (1:500, Nrf2; cat. no. ab62352; Abcam), nuclear receptor coactivator 4 (1:100, NCOA4; cat. no. ab62495; Abcam) and GADPH (1:1,000, cat. no. ab8245; Abcam) overnight at $4^{\circ} \mathrm{C}$. Subsequently, HRP-conjugated secondary antibody (HRP goat anti-rabbit, 1:20,000, cat. no. ab97051; HRP goat anti-mouse, cat. no. ab205719, 1:10,000) was incubated with the membranes at room temperature for $2 \mathrm{~h}$. ECL Plus Western Blotting Substrate (Pierce; Thermo Fisher Scientific, Inc.) was used to visualize protein bands, and ImageJ software (version $1.52 \mathrm{v}$; National Institutes of Health) was used for quantification.

Cell Counting Kit-8 (CCK-8) assay. Cell viability was determined using a CCK-8 assay. The cells were cultured in 96-well plates at a density of $1 \times 10^{5}$ cells/well. After treatment, CCK-8 reagent ( $10 \mu \mathrm{l}$; Beyotime Institute of Biotechnology) was added to the cells. After incubation for $2 \mathrm{~h}$, the absorbance of cells at a wavelength of $450 \mathrm{~nm}$ was determined in the different study groups using a microplate reader (Omega Bio-Tek, Inc.).

Statistical analysis. Data were processed using SPSS 18.0 software (SPSS, Inc.) and GraphPad Prism 8.0 (GraphPad Software, Inc.) and shown as the mean \pm SD. One-way ANOVA with Tukey's test was used for evaluating significant differences between groups. $\mathrm{P}<0.05$ was considered to indicate a statistically significant difference.

\section{Results}

ICA attenuates cell death and increases cell viability in LPS-induced synoviocytes. In the LPS group, cell viability significantly decreased (Fig. 1A) and cell death significantly increased compared with the control group (Fig. 1B and C), suggesting that the synovitis cell model was successfully established. Simultaneously, LPS-induced cell death was decreased by ICA and LPS-induced cell viability was increased by ICA in a concentration-dependent manner, suggesting that ICA has protective effects in LPS-induced synoviocytes.

ICA reduces MDA levels and iron content and increases GPX levels in a concentration-dependent manner in LPS-induced 

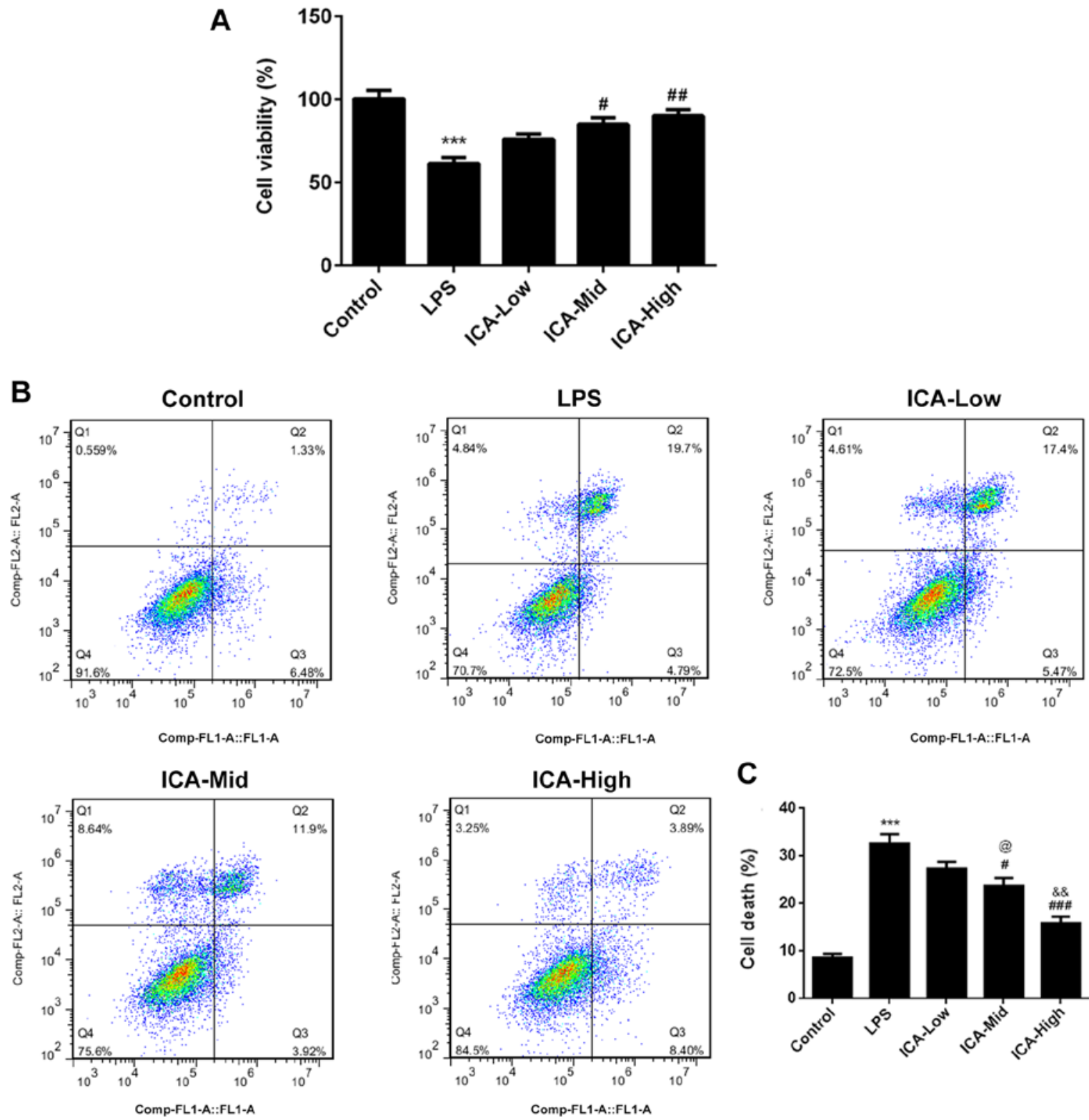

\section{C}

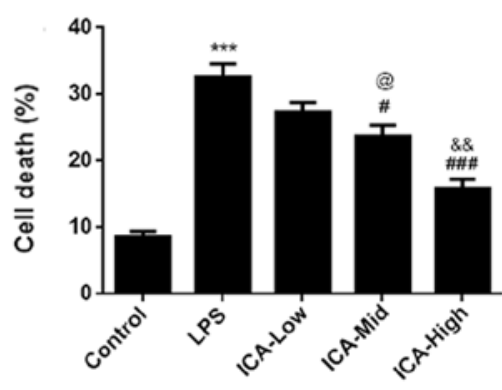

Figure 1. The effects of ICA on cell viability and death. (A) Cell viability and (B and C) cell death in the different study groups. Experimental data were obtained from five independent experiments and shown as the mean \pm standard deviation. ${ }^{* * *} \mathrm{P}<0.001$ vs. control; ${ }^{\#} \mathrm{P}<0.05$, ${ }^{\# \#} \mathrm{P}<0.01$ and ${ }^{\# \# \# P<0.001 ~ v s . ~ L P S}$; ${ }^{\circledR} \mathrm{P}<0.05$ vs. ICA-Low; ${ }^{\&} \mathrm{P}<0.01$ vs. ICA-Mid. ICA, icariin; ICA-Low, $2 \mu \mathrm{M}$ ICA; ICA-Mid, $5 \mu \mathrm{M}$ ICA; ICA-High, $10 \mu \mathrm{M}$ ICA; LPS, lipopolysaccharide.

synoviocytes. Lipid peroxidation is a type of oxidative stress and is a vital process in rheumatoid arthritis and osteoarthritis $(30,31)$. MDA, as the common biomarker of lipid peroxidation (32), was detected in the present study. There was a significant increase in MDA levels in the LPS group compared with the control (Fig. 2A), suggesting that lipid peroxidation levels are high in the synovitis cell model. MDA levels induced by LPS was reduced by MDA in a concentration-dependent manner, indicating that ICA has attenuative effects on lipid peroxidation in LPS-induced synoviocytes. Iron was confirmed as the stimulator of oxidative stress and iron overload $(33,34)$. In the present study, the iron content was significantly increased in the LPS group compared with the control group. The iron content induced by LPS was reduced by ICA in a concentration-dependent manner (Fig. 2B), demonstrating that ICA could attenuate the iron deposit in LPS-induced synoviocytes. GPX, an antioxidant enzyme (35), was also detected in the present study. GPX levels were significantly reduced by LPS compared with the control group. The levels of GPX induced by LPS was further increased by ICA in a concentration-dependent manner. These results suggested that ICA exerted antioxidant effects by reducing MDA levels and iron content and increasing GPX levels in LPS-induced synoviocytes.

ICA regulates the relative expressions level of ferroptosis proteins in LPS-induced synoviocytes. Lipid peroxidation and iron metabolism disorder, which are the major inducers of ferroptosis (36), both occurred in LPS-induced synoviocytes. Considering the attenuative effects of ICA on iron content and lipid peroxidation levels, it was speculated that there may be an association between ferroptosis and ICA. Xc-/GPX4, which is capable of suppressing lipid peroxidation, is a vital pathway for the inhibition of ferroptosis (37). In the current 
A

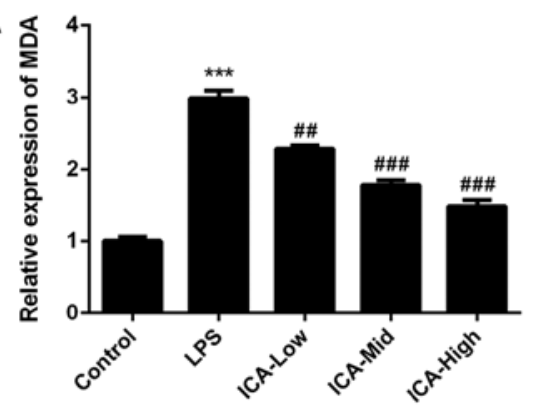

B

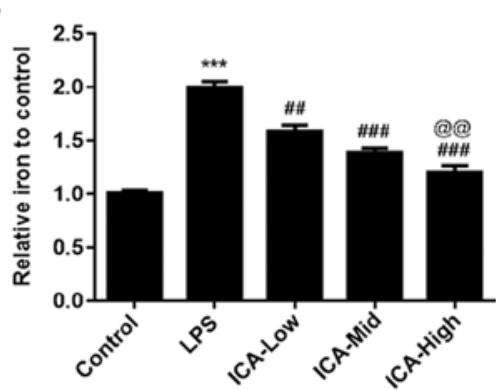

C

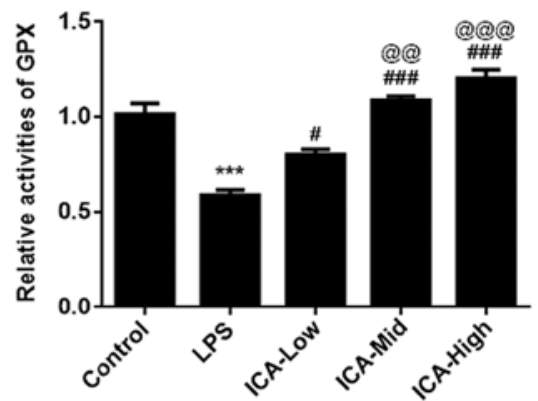

Figure 2. Effects of ICA on the levels of MDA, iron content and GPX. The levels of (A) MDA, (B) iron content and (C) GPX in the study groups Experimental data were obtained from five independent experiments and shown as the mean \pm standard deviation. ${ }^{@} \mathrm{P}<0.01$ and ${ }^{\circledR @ @ ~} \mathrm{P}<0.001 \mathrm{vs}$. ICA-Low; ${ }^{* * *} \mathrm{P}<0.001$ vs. control; ${ }^{\#} \mathrm{P}<0.05,{ }^{\# \#} \mathrm{P}<0.01$ and ${ }^{\# \# \#} \mathrm{P}<0.001$ vs. LPS. ICA, icariin; ICA-Low, $2 \mu \mathrm{M}$ ICA; ICA-Mid, $5 \mu \mathrm{M}$ ICA; ICA-High, $10 \mu \mathrm{M}$ ICA; LPS, lipopolysaccharide; MDA, malondialdehyde; GPX, glutathione peroxidase.
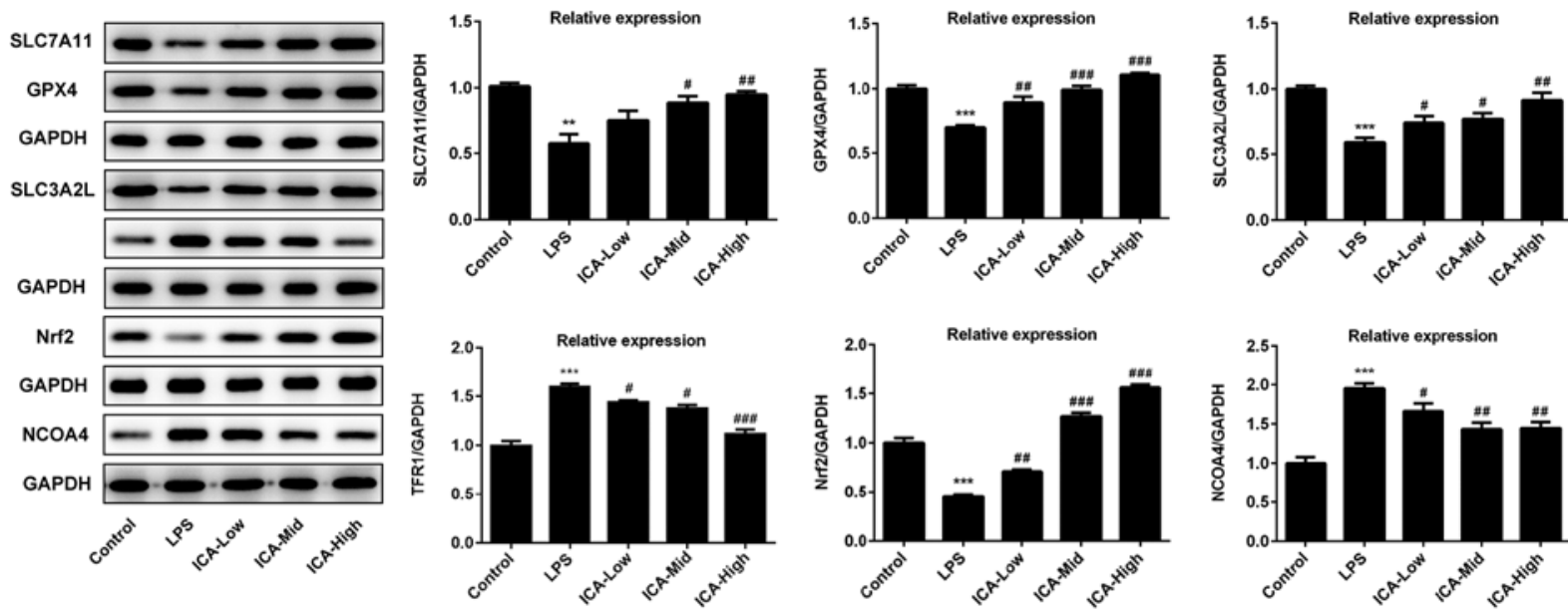

Figure 3. Effects of ICA on the levels of ferroptosis-associated proteins. The levels of proteins associated with ferroptosis in the study groups. The representative blot of five independent experiments with similar results are expressed as the mean \pm standard deviation. ${ }^{* * *} \mathrm{P}<0.001 \mathrm{vs}$. control; ${ }^{* * *} \mathrm{P}<0.01 \mathrm{vs}$. control; ${ }^{\# P}<0.05,{ }^{\# \#} \mathrm{P}<0.01$ and ${ }^{\# \# \#} \mathrm{P}<0.001$ vs. LPS. ICA, icariin; ICA-Low, $2 \mu \mathrm{M}$ ICA; ICA-Mid, $5 \mu \mathrm{M}$ ICA; ICA-High, $10 \mu \mathrm{M}$ ICA; LPS, lipopolysaccharide; SLC7A111, cystine/glutamate transporter; GPX, glutathione peroxidase; SLC3A2L, 4F2 cell-surface antigen heavy chain; TFR1, transferrin receptor protein 1; Nrf2, nuclear factor erythroid 2-related factor 2; NCOA4, nuclear receptor coactivator 4.

study, GPX4, SLC7A11 and SLC3A2L expression were all significantly decreased by LPS compared with controls, and this effect was further inhibited by ICA (Fig. 3), confirming that ICA inhibited ferroptosis via the activation of Xc-/GPX4. Pathways that are associated with iron metabolism were also investigated in the present study. TFR1, a vital importer for iron transport into the cells (38), was significantly upregulated by LPS compared with the control group, which is consistent with the high levels of iron content in the LPS group. The TFR1 levels induced by LPS was decreased by ICA in a concentration-dependent manner, suggesting that inhibition of TFR 1 is one of the avenues for the inhibitory effect of ICA on ferroptosis in LPS-induced synoviocytes. Nrf2 acts as a crucial factor for inhibition of ferroptosis (39), which was significantly reduced by LPS and further elevated by ICA in a concentration-dependent manner in LPS-induced cells. NCOA4 acts as mediator of ferritinophagy, resulting in iron accumulation and ferroptosis (40). As shown by the present data, NCOA4 levels were significantly upregulated in the LPS group compared with controls. Following ICA treatment, the NCOA4 levels induced by LPS gradually decreased upon increasing concentrations of ICA. Collectively, ICA inhibited ferroptosis via the activation of Xc-/GPX4 and Nrf2, and inhibition of TFR1 and NCOA4.

ICA attenuates cell death via inhibition of ferroptosis by activating the Xc-/GPX4 axis. In order to further investigate the underlying mechanism, the effects of ICA after blockage of Xc-/GPX4 axis with RSL3 was further assessed. RSL3 is known to be a type of ferroptosis activator (41). As shown by the data, the cell viability was significantly reduced and cell death was increased in the RSL3 group compared with the controls (Fig. 4A-C), confirming that marked ferroptotic cell death was observed after RSL3 induction. Iron and MDA content significantly increased and GPX levels significantly decreased in the RSL3 group compared with the ICA + RSL3 group (Fig. 5A-C). Thus, the effects of RSL on lipid peroxidation and iron content were counteracted by ICA and the effects of ICA in cells were achieved via activation of the Xc-/GPX4 axis. The expression of ferroptosis-associated proteins was also investigated. Compared with the ICA + RSL3 group, Xc-/GPX4-associated proteins (SLC7A11, GPX4 and SLC3A2L) and Nrf2 expression were significantly reduced by RSL3, demonstrating that Xc-/GPX4 and Nrf2 were activated 

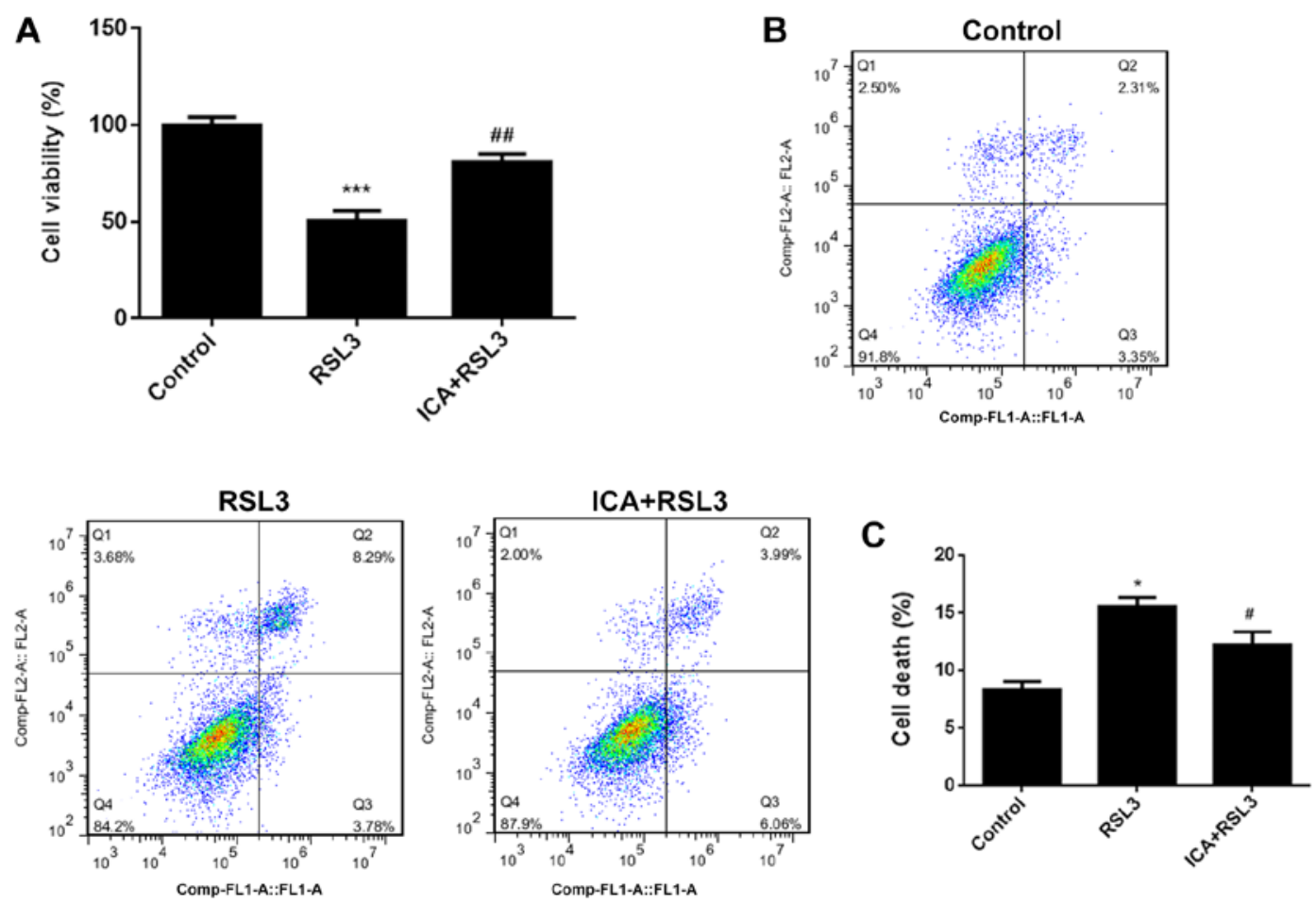

Figure 4. Effects of ICA on cell death and viability following inhibition of the Xc-/glutathione peroxidase 4 axis. (A) cell viability and (B and C) cell death in the different study groups. Experimental data were obtained from five independent experiments and shown as the mean \pm standard deviation. ${ }^{*} \mathrm{P}<0.01$ and ${ }^{* * * *} \mathrm{P}<0.001$ vs. control; ${ }^{\#} \mathrm{P}<0.05$ and ${ }^{\# \#} \mathrm{P}<0.01$ vs. RSL3. ICA, icariin.
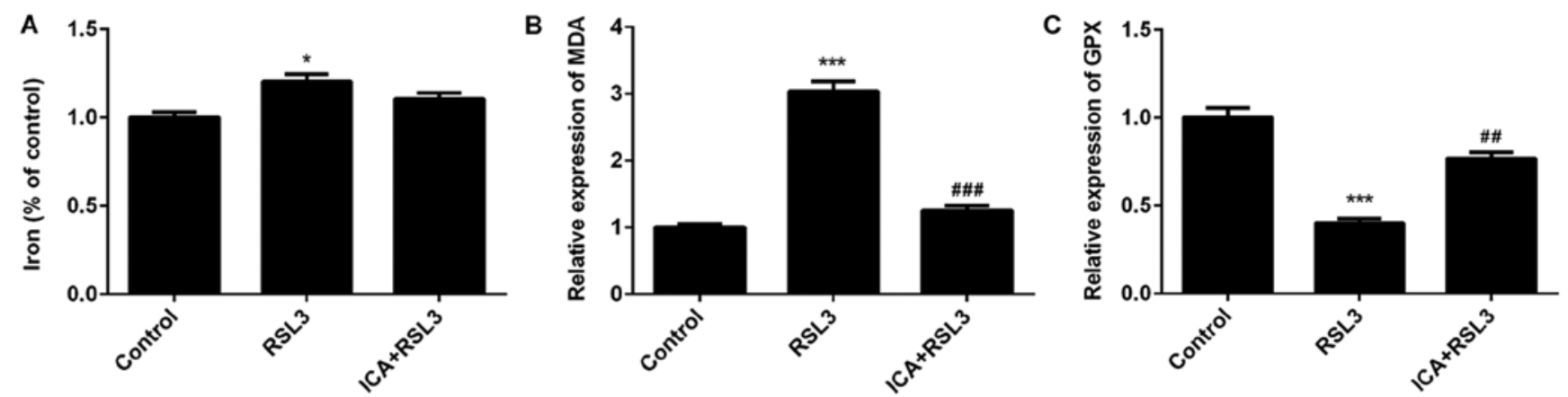

Figure 5. Effects of ICA on the levels of MDA, iron content and GPX following inhibition of the Xc-/GPX4 axis. The levels of (A) MDA, (B) iron content and (C) GPX in the study groups. Bar graphs were drawn from five independent experiments. Results were expressed as the mean \pm standard deviation. ${ }^{*} \mathrm{P}<0.05$ and ${ }^{* * *} \mathrm{P}<0.001$ vs. control; ${ }^{\# \#} \mathrm{P}<0.01$ and ${ }^{\# \# \#} \mathrm{P}<0.001$ vs. RSL3. ICA, icariin; MDA, malondialdehyde; GPX, glutathione peroxidase.

by ICA (Fig. 6). Following the blockage of the Xc-/GPX4 axis, there was almost no difference in the levels of NCOA4 and TFR1 between the ICA + RSL3 group and RSL3 group, suggesting that NCOA4 and TFR1 were not the major players in the mechanism of ICA. All these results support that the Xc-/GPX4 axis is involved in the effects of ICA via the modulation of ferroptosis.

\section{Discussion}

ICA has been demonstrated to serve a vital role in both rheumatoid arthritis and osteoarthritis and reported to act as a regulator of gene expression and cellular functions in the synoviocytes of osteoarthritis $(19,42)$. ICA reduces the number of Th17 cells, resulting in repression of the generation of IL-1 in rheumatoid arthritis (23). Synovitis is the vital pathological process for arthritis and osteoarthritis (13). The mechanism and effects of ICA in synovitis is still not fully elaborated. In the present study, it was found that ICA protected synoviocytes from death via the modulation of ferroptosis.

Ferroptosis was confirmed to be involved in several pathological processes. Ferroptosis is confirmed as the novel mechanism of anticancer agents, such as non-small cell lung cancer and breast cancer (43). Edaravone functions in amyotrophic lateral sclerosis via the inhibition of ferroptosis (44). Ferroptosis, which contributes to the progress of ulcerative colitis, is a promising treatment target for ulcerative colitis (45). However, whether ferroptosis is involved in osteoarthritis or rheumatoid arthritis is still unknown. Lipid peroxidation and abnormal iron metabolism, as critical stimulators for ferroptosis, are involved in osteoarthritis and rheumatoid arthritis (46-50). Thus, it was postulated that ferroptosis may 

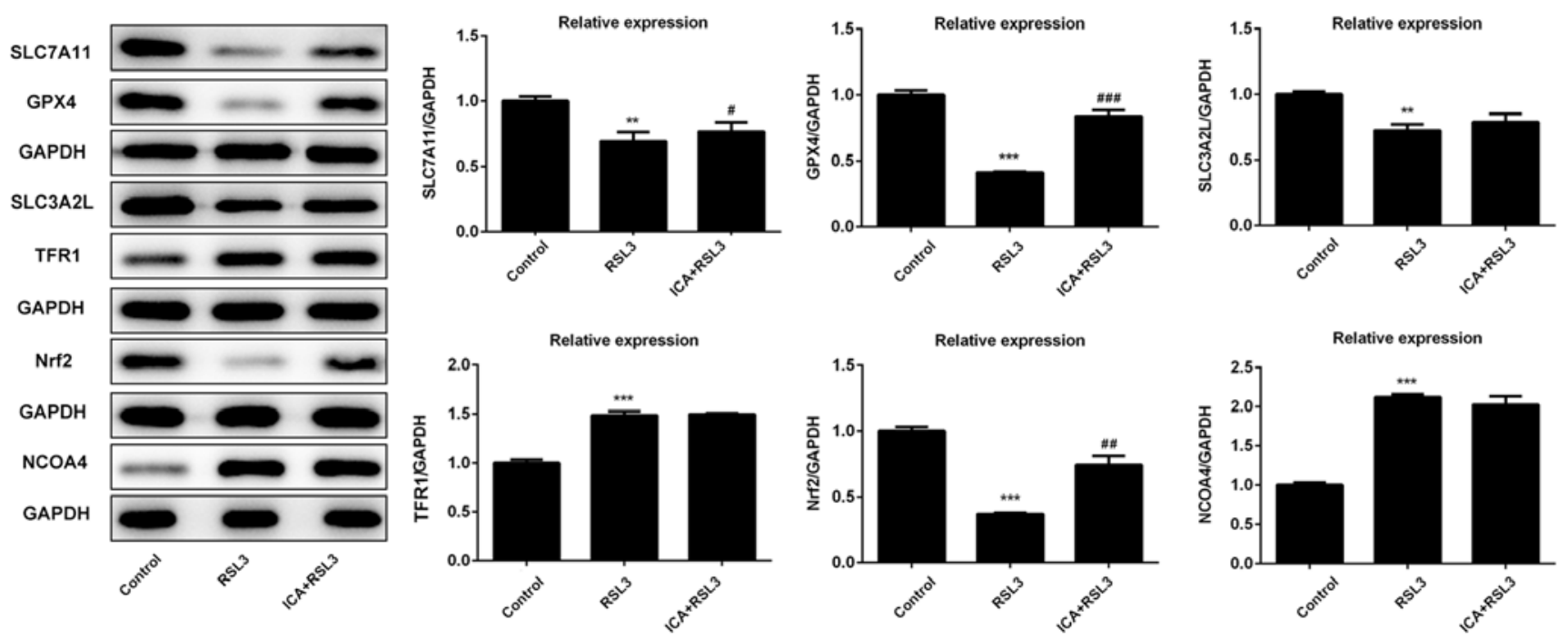

Figure 6. Effects of ICA on ferroptosis-associated protein expression following inhibition of the Xc-/GPX4 axis. The expression levels of ferroptosis-associated proteins in the study groups. The protein levels been standardized against GAPDH expression. Values are expressed as the mean \pm standard deviation. ${ }^{* *} \mathrm{P}<0.01$ and ${ }^{* * *} \mathrm{P}<0.001$ vs. control; ${ }^{\#} \mathrm{P}<0.05$, ${ }^{\# \#} \mathrm{P}<0.01$ and ${ }^{\# \# \#} \mathrm{P}<0.001$ vs. RSL3. ICA, icariin; SLC7A111, cystine/glutamate transporter; GPX, glutathione peroxidase; SLC3A2L, 4F2 cell-surface antigen heavy chain; TFR1, transferrin receptor protein 1; Nrf2, nuclear factor erythroid 2-related factor 2; NCOA4, nuclear receptor coactivator 4 .

be also be involved in synovitis, which is the vital process of osteoarthritis and rheumatoid.

As ferroptosis is a form of inducing cell death, the cell viability was evaluated in the present study. It was found that the cell viability was significantly reduced in the cell model of LPS-induced synoviocytes, which is further confirmed by cell death observed in the LPS group. The cell death induced by LPS was significantly reduced by ICA at a high concentration. The lipid peroxidation and iron content levels were detected to determine whether the cell death in the LPS group was induced by ferroptosis. MDA, as a common indicator of lipid peroxidation, is increased in synoviocytes with osteoarthritis and rheumatoid arthritis $(51,52)$. Simultaneously, GPX, an anti-oxidant agent, is decreased in osteoarthritis and rheumatoid arthritis $(53,54)$. Consistent with previous studies, MDA levels were increased and GPX levels were decreased in the LPS group. Furthermore, it was found that ICA reversed the effects on MDA and GPX levels induced by LPS in a concentration-dependent manner. Abnormal iron metabolism is another contributor of ferroptosis and iron deposits are found in osteoarthritis and rheumatoid arthritis $(50,55,56)$. In accordance with previous studies, it was found that the iron content was increased in the LPS group. In addition, ICA reduced the iron content induced by LPS. Collectively, the data demonstrated that ferroptosis may be involved in synovitis and ICA exerted inhibitory effects on ferroptosis.

To further confirm the results observed, ferroptosis-associated proteins were detected in the present study. The Xc-/GPX4 axisregulated lipid ROS levels and its inhibition contributed to ferroptosis (57). In the present study, the levels of GPX4, SLC7A11 and SLC3A2L were decreased in the LPS group and this effect was inhibited by ICA, suggesting that ferroptosis was involved in synovitis and ICA inhibited ferroptosis. TFR 1 and NCOA4, as contributors of ferroptosis were increased by LPS, and Nrf2 was reduced by LPS, confirming that ferroptosis was involved in synovitis. Furthermore, the effects of LPS on the levels of TFR1, NCOA4 and Nrf2 were all alleviated by ICA via a concentration-dependent manner, further supporting that ICA has suppressive effects on ferroptosis.

RSL3, a GPX4 inhibitor (58), was used to investigate whether the Xc-/GPX4 axis was the main avenue for the inhibitory effects of ICA on ferroptosis. As expected, cell viability was decreased and cell death was increased by RSL3, suggesting that following inhibition of the Xc-/GPX4 axis, ferroptosis was enhanced. Lipid peroxidation and iron content were elevated by RSL3. Moreover, the aforementioned effects of RSL3 were reversed by ICA, confirming that ICA exerted inhibitory effects on ferroptosis via activation of the Xc-/GPX4 axis. GPX4, SLC7A11 and SLC3A2L were all reduced by RSL3 and this effect was partly reversed by ICA, strongly supporting that the protective effects of ICA on cell viability was mediated via activation of the Xc-/GPX4 axis, which protects against ferroptosis. ICA has no effects on the levels of TFR1, NCOA4 and SLC3A2L induced by RSL3, indicating that ICA exerted anti-ferroptosis mainly depending on SLC7A11-/GPX4 axis. Thus, the results confirm that the $\mathrm{Xc}-/ \mathrm{GPX} 4$ axis is a mediator for the protective effects of ICA in synovitis.

To the best of our knowledge, the present study first identified that ferroptosis was involved in synovitis and ICA exerted protective effects via inhibition of ferroptosis and by the activation of the Xc-/GPX4 axis in synovitis, providing a new strategy for the treatment of synovitis. However, the ferroptosis regulation of ICA via Xc-/GPX4 axis in osteoarthritis needs to be further confirmed through in vivo study. Determining how other ferroptosis activator or inhibitors affect ferroptosis of osteoarthritis would be helpful to further understand the mechanism of ICA in ferroptosis.

\section{Acknowledgements}

Not applicable. 


\section{Funding}

Not applicable.

\section{Availability of data and materials}

The datasets used and/or analyzed during the current study are available from the corresponding author on reasonable request.

\section{Authors' contributions}

HL and RZ conceived and designed the study, collected, analysed and interpreted the data, and revised the manuscript. HL wrote the manuscript. All authors read and approved the final manuscript.

\section{Ethics approval and consent to participate}

Not applicable.

\section{Patient consent for publication}

Not applicable.

\section{Competing interests}

The authors declare that they have no competing interests.

\section{References}

1. Smolen JS, Aletaha D and McInnes IB: Rheumatoid arthritis. Lancet 388: 2023-2038, 2016.

2. Safiri S, Kolahi AA, Hoy D, Smith E, Bettampadi D, Mansournia MA, Almasi-Hashiani A, Ashrafi-Asgarabad A Moradi-Lakeh M, Qorbani M, et al: Global, regional and national burden of rheumatoid arthritis 1990-2017: A systematic analysis of the global burden of disease study 2017. Ann Rheum Dis 78 1463-1471, 2019.

3. Smolen JS, Aletaha D, Barton A, Burmester GR, Emery P, Firestein GS, Kavanaugh A, McInnes IB, Solomon DH, Strand V and Yamamoto K: Rheumatoid arthritis. Nat Rev Dis Primers 4 18001,2018

4. Orsolini G, Fassio A, Rossini M, Adami G, Giollo A, Caimmi C Idolazzi L, Viapiana O and Gatti D: Effects of biological and targeted synthetic DMARDs on bone loss in rheumatoid arthritis. Pharmacol Res 147: 104354, 2019.

5. Lazzerini PE, Capecchi PL and Laghi-Pasini F: Systemic inflammation and arrhythmic risk: Lessons from rheumatoid arthritis Eur Heart J 38: 1717-1727, 2017.

6. Li Y, Jiang L, Zhang Z, Li H, Jiang L, Wang L and Li Z: Clinical characteristics of rheumatoid arthritis patients with peripheral neuropathy and potential related risk factors. Clin Rheumatol 38 2099-2107, 2019.

7. Scott DL, Wolfe F and Huizinga TWJ: Rheumatoid arthritis. Lancet 376: 1094-1108, 2010

8. Lundberg K, Bengtsson C, Kharlamova N, Reed E, Jiang X, Kallberg H, Pollak-Dorocic I, Israelsson L, Kessel C, Padyukov L, et al: Genetic and environmental determinants for disease risk in subsets of rheumatoid arthritis defined by the anticitrullinated protein/peptide antibody fine specificity profile. Ann Rheum Dis 72: 652-658, 2013.

9. McInnes IB and Schett G: The pathogenesis of rheumatoid arthritis. N Engl J Med 365: 2205-2219, 2011.

10. Falconer J, Murphy AN, Young SP, Clark AR, Tiziani S, Guma M and Buckley CD: Review: Synovial cell metabolism and chronic inflammation in rheumatoid arthritis. Arthritis Rheumatol 70 984-999, 2018

11. Chen X, Zhang L, Song Q and Chen Z: MicroRNA-216b regulates cell proliferation, invasion and cycle progression via interaction with cyclin T2 in gastric cancer. Anticancer Drugs 31: 623-631, 2020.
12. Bragg R, Gilbert W, Elmansi AM, Isales CM, Hamrick MW, Hill WD and Fulzele S: Stromal cell-derived factor-1 as a potential therapeutic target for osteoarthritis and rheumatoid arthritis. Ther Adv Chronic Dis 10: 2040622319882531, 2019.

13. Mathiessen A and Conaghan PG: Synovitis in osteoarthritis: Current understanding with therapeutic implications. Arthritis Res Ther 19: 18, 2017.

14. Scanzello CR and Goldring SR: The role of synovitis in osteoarthritis pathogenesis. Bone 51: 249-257, 2012.

15. Tan HL, Chan KG, Pusparajah P, Saokaew S, Duangjai A, Lee LH and Goh BH: Anti-cancer properties of the naturally occurring aphrodisiacs: Icariin and its derivatives. Front Pharmacol 7: 191, 2016.

16. Zhang B, Wang G, He J, Yang Q, Li D, Li J and Zhang F: Icariin attenuates neuroinflammation and exerts dopamine neuroprotection via an Nrf2-dependent manner. J Neuroinflammation 16: 92 , 2019.

17. Zheng Y, Zhu G, He J, Wang G, Li D and Zhang F: Icariin targets Nrf2 signaling to inhibit microglia-mediated neuroinflammation. Int Immunopharmacol 73: 304-311, 2019.

18. Wang GQ, Li DD, Huang C, Lu DS, Zhang C, Zhou SY, Liu J and Zhang F: Icariin reduces dopaminergic neuronal loss and microglia-mediated inflammation in vivo and in vitro. Front Mol Neurosci 10: 441, 2018.

19. Zu Y, Mu Y, Li Q, Zhang ST and Yan HJ: Icariin alleviates osteoarthritis by inhibiting NLRP3-mediated pyroptosis. J Orthop Surg Res 14: 307, 2019.

20. Huang H, Zhang ZF, Qin FW, Tang W, Liu DH, Wu PY and Jiao F: Icariin inhibits chondrocyte apoptosis and angiogenesis by regulating the TDP-43 signaling pathway. Mol Genet Genomic Med 7: e00586, 2019.

21. Wang P, Xiong X, Zhang J, Qin S, Wang W and Liu Z: Icariin increases chondrocyte vitality by promoting hypoxia-inducible factor-1 $\alpha$ expression and anaerobic glycolysis. Knee 27: 18-25, 2020.

22. Shen R and Wang JH: The effect of icariin on immunity and its potential application. Am J Clin Exp Immunol 7: 50-56, 2018.

23. Chi L, Gao W, Shu X and Lu X: A natural flavonoid glucoside, icariin, regulates Th17 and alleviates rheumatoid arthritis in a murine model. Mediators Inflamm 2014: 392062, 2014.

24. Cheleschi S, Gallo I, Barbarino M, Giannotti S, Mondanelli N, Giordano A, Tenti S and Fioravanti A: MicroRNA mediate visfatin and resistin induction of oxidative stress in human osteoarthritic synovial fibroblasts via NF- $\kappa \mathrm{B}$ pathway. Int J Mol Sci 20: 5200, 2019 .

25. Franz A, Joseph L, Mayer C, Harmsen JF, Schrumpf H, Frobel J, Ostapczuk MS, Krauspe R and Zilkens C: The role of oxidative and nitrosative stress in the pathology of osteoarthritis: Novel candidate biomarkers for quantification of degenerative changes in the knee joint. Orthop Rev (Pavia) 10: 7460, 2018.

26. Ostalowska A, Birkner E, Wiecha M, Kasperczyk S, Kasperczyk A, Kapolka D and Zon-Giebel A: Lipid peroxidation and antioxidant enzymes in synovial fluid of patients with primary and secondary osteoarthritis of the knee joint. Osteoarthritis Cartilage 14: 139-145, 2006.

27. Xie Y, Hou W, Song X, Yu Y, Huang J, Sun X, Kang R and Tang D: Ferroptosis: Process and function. Cell Death Differ 23: 369-379, 2016.

28. Zhang M, Liu J, Guo W, Liu X, Liu S and Yin H: Icariin regulates systemic iron metabolism by increasing hepatic hepcidin expression through Stat 3 and Smad1/5/8 signaling. Int J Mol Med 37: 1379-1388, 2016

29. Yin S, Wang P, Xing R,ZhaoL,LiX,Zhang L and Xiao Y: Transient receptor potential ankyrin 1 (TRPA1) mediates lipopolysaccharide (LPS)-induced inflammatory responses in primary human osteoarthritic fibroblast-like synoviocytes. Inflammation 41: 700-709, 2018

30. Tsikas D: Assessment of lipid peroxidation by measuring malondialdehyde (MDA) and relatives in biological samples: Analytical and biological challenges. Anal Biochem 524: 13-30, 2017.

31. Srivastava NK, Sharma S, Sinha N, Mandal SK and Sharma D: Abnormal lipid metabolism in a rat model of arthritis: One possible pathway. Mol Cell Biochem 448: 107-124, 2018.

32. Luczaj W, Jarocka-Karpinska I, Sierakowski S, Andrisic L, Zarkovic $\mathrm{N}$ and Skrzydlewska E: Lipid peroxidation in Rheumatoid arthritis; consequences and monitoring. Free Radic Biol Med 75 (Suppl 1): S49, 2014.

33. Winyard PG, Blake DR, Chirico S, Gutteridge JM and Lunec J: Mechanism of exacerbation of rheumatoid synovitis by total-dose iron-dextran infusion: In-vivo demonstration of iron-promoted oxidant stress. Lancet 1: 69-72, 1987. 
34. Morris CJ, Wainwright AC, Steven MM and Blake DR: The nature of iron deposits in haemophilic synovitis. An immunohistochemical, ultrastructural and X-ray microanalytical study. Virchows Arch A Pathol Anat Histopathol 404: 75-85, 1984.

35. Zhang C, Zhang W, Shi R, Tang B and Xie S: Coix lachryma-jobi extract ameliorates inflammation and oxidative stress in a complete Freund's adjuvant-induced rheumatoid arthritis model. Pharm Biol 57: 792-798, 2019.

36. Yang L, Wang H, Yang X, Wu Q, An P, Jin X, Liu W, Huang X, $\mathrm{Li}$ Y, Yan S, et al: Auranofin mitigates systemic iron overload and induces ferroptosis via distinct mechanisms. Signal Transduct Target Ther 5: 138, 2020.

37. Yang WS and Stockwell BR: Ferroptosis: Death by lipid peroxidation. Trends Cell Biol 26: 165-176, 2016.

38. Kawabata H: Transferrin and transferrin receptors update. Free Radic Biol Med 133: 46-54, 2019.

39. Dodson M, Castro-Portuguez R and Zhang DD: NRF2 plays a critical role in mitigating lipid peroxidation and ferroptosis. Redox Biol 23: 101107, 2019.

40. Zhou B, Liu J, Kang R, Klionsky DJ, Kroemer G and Tang D: Ferroptosis is a type of autophagy-dependent cell death. Semin Cancer Biol 66: 89-100, 2020.

41. Imai H, Matsuoka M, Kumagai T, Sakamoto T and Koumura T: Lipid peroxidation-dependent cell death regulated by GPx4 and ferroptosis. Curr Top Microbiol Immunol 403: 143-170, 2017.

42. Pan L, Zhang Y, Chen N and Yang L: Icariin regulates cellular functions and gene expression of osteoarthritis patient-derived human fibroblast-like synoviocytes. Int J Mol Sci 18: 2656 , 2017.

43. Su Y, Zhao B, Zhou L, Zhang Z, Shen Y, Lv H, AlQudsy LH and Shang P: Ferroptosis, a novel pharmacological mechanism of anti-cancer drugs. Cancer Lett 483: 127-136, 2020.

44. Spasić S, Nikolić-Kokić A, Miletić S, Oreščanin-Dušić Z, Spasić MB, Blagojević D and Stević Z: Edaravone may prevent ferroptosis in ALS. Curr Drug Targets 21: 776-780, 2020.

45. Xu M, Tao J, Yang Y, Tan S, Liu H, Jiang J, Zheng F and Wu B Ferroptosis involves in intestinal epithelial cell death in ulcerative colitis. Cell Death Dis 11: 86, 2020.

46. Łuczaj W, Gindzienska-Sieskiewicz E, Jarocka-Karpowicz I, Andrisic L, Sierakowski S, Zarkovic N, Waeg G and Skrzydlewska E: The onset of lipid peroxidation in rheumatoid arthritis: Consequences and monitoring. Free Radic Res 50: 304-313, 2016.
47. Olszewska-Slonina DM, Jung S, Olszewski KJ, Cwynar A and Drewa G: Evaluation of selected parameters of lipid peroxidation and paraoxonase activity in blood of patients with joint osteoarthritis. Protein Pept Lett 25: 853-861, 2018.

48. Morris CJ, Blake DR, Wainwright AC and Steven MM: Relationship between iron deposits and tissue damage in the synovium: An ultrastructural study. Ann Rheum Dis 45: 21-26, 1986.

49. Ogilvie-Harris DJ and Fornaiser VL: Synovial iron deposition in osteoarthritis and rheumatoid arthritis. J Rheumatol 7: 30-36, 1980.

50. Bennett RM, Williams ED, Lewis SM and Holt PJ: Synovial iron deposition in rheumatoid arthritis. Arthritis Rheum 16: 298-304, 1973.

51. Grigolo B, Roseti L, Fiorini M and Facchini A: Enhanced lipid peroxidation in synoviocytes from patients with osteoarthritis. J Rheumatol 30: 345-347, 2003.

52. Grönwall C, Amara K, Hardt U, Krishnamurthy A, Steen J, Engström M, Sun M, Ytterberg AJ, Zubarev RA, ScheelToellnerD,etal: Autoreactivity to malondialdehyde-modifications in rheumatoid arthritis is linked to disease activity and synovial pathogenesis. J Autoimmun 84: 29-45, 2017.

53. Surapneni KM and Chandrasada Gopan VS: Lipid peroxidation and antioxidant status in patients with rheumatoid arthritis. Indian J Clin Biochem 23: 41-44, 2008.

54. Sutipornpalangkul W, Morales NP, Charoencholvanich K and Harnroongroj T: Lipid peroxidation, glutathione, vitamin E, and antioxidant enzymes in synovial fluid from patients with osteoarthritis. Int J Rheum Dis 12: 324-328, 2009.

55. Fritz P, Saal JG, Wicherek C, König A, Laschner W and Rautenstrauch H: Quantitative photometrical assessment of iron deposits in synovial membranes in different joint diseases. Rheumatol Int 15: 211-216, 1996.

56. Bennett RM: Synovial iron deposition in osteoarthritis and rheumatoid arthritis. J Rheumatol 7: 583, 1980.

57. Cao JY and Dixon SJ: Mechanisms of ferroptosis. Cell Mol Life Sci 73: 2195-2209, 2016.

58. Shin D, Kim EH, Lee J and Roh JL: Nrf2 inhibition reverses resistance to GPX4 inhibitor-induced ferroptosis in head and neck cancer. Free Radic Biol Med 129: 454-462, 2018. 